SHORT REPORT

\title{
Improving hearing assessment of children post-meningitis
}

\author{
C Wilson, A Roberts, D Stephens
}

Arch Dis Child 2003;88:976-977

The improvement in audiological testing of children postmeningitis with one retrospective and two prospective audit cycles, is described. Direct referral by telephone prior to discharge and liaison with public health colleagues resulted in an increased assessment from $50 \%$ to $99 \%$ and $89 \%$ in successive audits. The proportion seen within six weeks also improved dramatically.

$\mathrm{H}$ earing impairment has significant consequences for a child's communication, educational achievement, and emotional and social wellbeing. ${ }^{1}$ Every year in the UK one in every 1000 children are born with a permanent hearing impairment. ${ }^{2}$ A further $50-90 \%$ more children acquire a hearing impairment, of which meningitis is the most common cause. ${ }^{34}$

Early assessment of hearing in children after meningitis is recommended. ${ }^{56}$ Early identification is essential for successful habilitation, including cochlear implantation. The cochlear duct can be obliterated by osteoneogenesis within a few months of meningitis, which may make implantation ineffective, or impossible. ${ }^{7}$

Many studies have shown that difficulties exist in ensuring all children have hearing assessment after meningitis, with reports of between $7.7 \%$ and $27 \%$ of children not being tested..$^{8-10}$

\section{METHODS}

A retrospective audit of case notes of children notified to the Public Health Department as having meningitis over a 12 month period (June 1994 to May 1995) was carried out in 1995. Prior to this audit there were no formal guidelines for the audiological testing of children post-meningitis. The interventions introduced were reaudited in two further prospective audits (January 1998 to December 1999, and January to December 2001).

Data from the International Classification of Disease (ICD $)^{11}$ was used to identify children coded with a discharge diagnosis of meningitis to determine whether all cases had been referred for assessment. Random sampling of case notes was also performed.

\section{RESULTS}

\section{First audit (1994-95) - retrospective}

Of the 26 cases notified, $13(50 \%)$ were assessed, and eight $(31 \%)$ were tested within eight weeks. Seven had no record of hearing assessment, and in six cases no information was available.

\section{Outcome of first audit}

- Implementation of direct referral by paediatric ward staff (by telephone) prior to discharge.

- To aim to test all children within six weeks of discharge.

\section{Second audit cycle (1998-99)-prospective}

Eighty seven children were referred for assessment; 86 (99\%) children were assessed, of whom 80 (93\%) were tested within six weeks.

Of the 62 children who had an ICD discharge code of meningitis in that period, only 28 had been referred for audiological assessment.

\section{Outcome of second audit}

- Liaison with Public Health introduced to provide a "safety net".

\section{Third audit cycle (2001)-prospective}

Sixty one children referred; 54 (89\%) assessed, all within nine weeks, 49 (80\%) within six weeks. Table l summarises the breakdown of referral pattern and time of testing.

Examination of the ICD coding and case notes still showed not all children were referred for audiological assessment as illustrated in table 2 . Of the nine children referred by Public Health (and not by the ward), examination of case notes and ICD coding found: four possible meningitis, one meningococcal infection, three viral illnesses, one no information available.

\section{Outcome of third audit}

- Improved communication with the referring paediatrician and primary care team to reduce number of non-attenders.

- An information leaflet on hearing impairment postmeningitis (developed by the Meningitis Trust) to be sent to all families who fail to attend/decline assessment.

- An updated referral pathway to be included in the Paediatric Houseman's Handbook (distributed to all paediatric junior staff within the trust).

- Liaison with intensive care staff to highlight the importance of hearing assessment when planning discharge.

\section{DISCUSSION}

Our data show that it is possible to ensure that the majority of children referred have hearing assessment post-meningitis. This was achieved by raising the awareness of the quality standard for hearing assessment and developing local achievable standards within a multidisciplinary framework. We feel that the introduction of liaison with Public Health provides an important "safety net".

However, difficulty exists in identifying all children with meningitis, even using ICD codes and public health notifications. A criticism of this paper is that we are still unsure exactly how many children had meningitis and therefore what proportion were referred for assessment. Furthermore, the sources of data in the retrospective audit (public health records) are different to that from the two prospective audits (audiology records), which may affect comparability of results.

Some children referred were not tested, particularly if not referred from the ward prior to discharge. Testing of children with otoacoustic emissions prior to discharge from hospital 
Table 1 Breakdown of referral pattern and time of testing from discharge during the period JanuaryDecember 2001 (third audit cycle)

\begin{tabular}{lll}
\hline Place of referral & Number of children & Time of audiological testing \\
\hline Ward & 50 & 47 within 6 weeks \\
& 9 & 50 within 9 weeks \\
Public Health & 2 within 6 weeks \\
& 2 within 8 weeks \\
& 4 parents declined testing \\
& & 1 DNA \\
Paediatric & 2 & 2 DNA \\
outpatients & & \\
\hline
\end{tabular}

has been advocated. ${ }^{12}$ At present equipment for such testing is not fully available in our centres.

Consideration must be given to definition of cases; children with viral meningitis were not referred, as there are few reports of sensorineural hearing impairment in this condition. ${ }^{13}$ However, cases of meningococcal septicaemia were referred for assessment, as involvement of the meninges could not be excluded.

Finally, as shown in this audit, and other studies ${ }^{8}$ a well defined referral pathway can improve assessment rates postmeningitis. This paper also highlights the importance of audit and intervention.

\section{ACKNOWLEDGEMENTS}

We would like to thank Wendy Rabiotti and Sara Hillier (paediatric audiology department), Angela Ingram (quality and clinical audit facilitator), Liz Heal (information department), and Dr M Lyons and Jackie Murray (Public Health) for their help and support.

\section{Authors' affiliations}

C Wilson, A Roberts, D Stephens, Welsh Hearing Institute, University of Wales College of Medicine, Cardiff, UK

Correspondence to: Dr D Stephens, Welsh Hearing Institute, University of Wales College of Medicine, Cardiff CF14 4XW, UK; stephensd@cardiff.ac.uk

Accepted 28 February 2003
Table 2 Reasons for the non-referral of children with a discharge diagnosis coding of meningitis (third audit cycle)

\begin{tabular}{ll}
\hline Number of children & Reason for non-referral \\
\hline 1 & Died \\
4 & Discharged to referring hospital \\
4 & Viral meningitis \\
2 & Oncology patients already known to audiology \\
1 & Meningococcal hip infection \\
1 & Encephalomyelitis \\
3 & Notes not available \\
\hline
\end{tabular}

\section{REFERENCES}

1 Davis A Bamford J, Wilson I, et al. A critical review of the role of universal neonatal hearing screening in the detection of congenital hearing impairment. Health Technology Assessment 1997;1(10).

2 Watkin PM, Baldwin M. Confirmation of deafness in infancy. Arch Dis Child 1999:81:380-9.

3 Fortnum HM, Summerfield AQ, Marshall DH, et al. Prevalence of permanent hearing impairment in the United Kingdom and implications for universal neonatal hearing screening: questionnaire based ascertainment study. BMJ 2001;323:536-40.

4 Davis A, Wood S. The epidemiology of childhood hearing impairment: factors relevant to planning of services. Br J Audiol 1992;26:77-90.

5 Hall DM. Health for all children. In: A programme for child health surveillance, 2nd edn. Oxford: Oxford University Press, 1991.

6 National Deaf Children's Society. Quality Standard in Paediatric Audiology, Vol 1: Guidelines for the early identification of hearing impairment. London: National Deaf Children's Society, 1994.

7 Dodds A, Tyszkiewicz E, Ramsden R. Cochlear implantation after bacterial meningitis: the dangers of delay. Arch Dis Child 1997;76:139-40.

8 Roirdan A, Thompson A, Hodgson J. Hearing assessment after meningitis and meningococcal disease. Arch Dis Child 1995:72:441-2.

9 Drake R, Dravitski J, Voss L. Hearing in children after meningococcal meningitis. J Paediatr Child Health 2000;36:240-3.

10 Bedford H, de Louvois J, Halket S, et al. Meningitis in infancy in England and Wales: follow up at age 5 years. BMJ 2001:323:533-6.

11 WHO. International Statistical Classification of Diseases and related health problems (ICD-10). 10th revision. Geneva: World Health Organisation, 1992.

12 Richardson MP, Williamson TJ, Reid A, et al. Otoacoustic emissions as a screening test for hearing impairment in children recovering from acute bacterial meningitis. Pediatrics 1998;6:1364-8.

13 Fortnum H, Davis A. Hearing impairment in children after bacterial meningitis; a review. Arch Dis Child 1992;67:1128-33. 